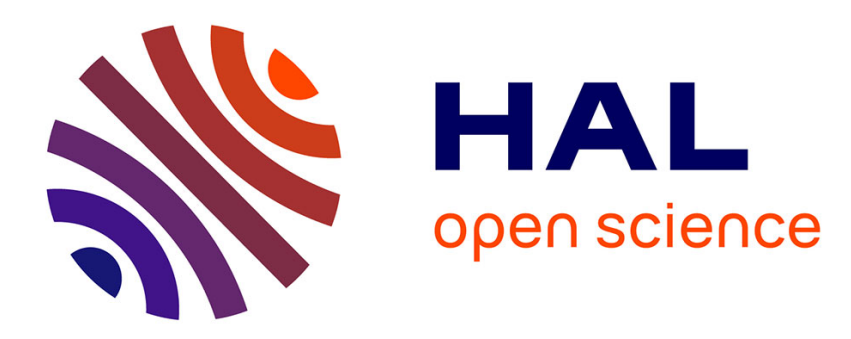

\title{
Atteinte rénale au cours du syndrome de Sjögren
}

Xavier Mariette, Hélène Francois

\section{To cite this version:}

Xavier Mariette, Hélène Francois. Atteinte rénale au cours du syndrome de Sjögren . Néphrologie \&

Thérapeutique, 2020, 16 (7), pp.440-452. 10.1016/j.nephro.2020.10.002 . hal-03158704

\section{HAL Id: hal-03158704 \\ https: / hal.sorbonne-universite.fr/hal-03158704}

Submitted on 4 Mar 2021

HAL is a multi-disciplinary open access archive for the deposit and dissemination of scientific research documents, whether they are published or not. The documents may come from teaching and research institutions in France or abroad, or from public or private research centers.
L'archive ouverte pluridisciplinaire HAL, est destinée au dépôt et à la diffusion de documents scientifiques de niveau recherche, publiés ou non, émanant des établissements d'enseignement et de recherche français ou étrangers, des laboratoires publics ou privés. 


\title{
Atteinte rénale au cours du syndrome de Sjögren *
}

\author{
Hélène FRANÇOIS ${ }^{a *}$, Xavier MARIETTE ${ }^{b, c}$
}

a Département d’Urgences Néphrologiques et Transplantation Rénale, , Hôpital Tenon, AP-HP, Sorbonne Université, Paris, France

${ }^{\mathrm{b}}$ Université Paris-Saclay, INSERM, CEA, Centre de recherche en Immunologie des infections virales et des maladies auto-immunes ; AP-HP.Université Paris-Saclay, Hôpital Bicêtre, Rheumatology department, 94270, Le Kremlin Bicêtre, France.

* Auteur correspondant ; e-mail : helene.francois@aphp.fr

${ }^{\star}$ Cet article est paru initialement dans EMC - Néphrologie 2017;14(3):1-11 [article 18-040-L-15]. Nous remercions la rédaction d'EMC-Néphrologie pour son aimable autorisation de reproduction.

\section{Résumé}

Le syndrome de Sjögren primitif est une maladie auto-immune caractérisée par une infiltration lymphoplasmocytaire des glandes exocrines (salivaires et lacrymales) entraînant le symptôme cardinal qui est un syndrome sec buccal et oculaire. Les complications systémiques sont possibles mais l'atteinte rénale est rare et affecte en général moins de $10 \%$ des patients. L'atteinte rénale la plus spécifique et fréquente au cours du syndrome de Sjögren primitif est la néphrite tubulo-interstitielle au cours de laquelle l'interstitium est infiltré par des lymphocytes et des plasmocytes de façon similaire à ce qui est observé dans les glandes exocrines. Des troubles hydroélectrolytiques peuvent survenir comme l'acidose tubulaire distale, le diabète insipide, le syndrome de Gitelman ou de Fanconi. L'atteinte glomérulaire est moins fréquente et correspond à une glomérulonéphrite membranoproliférative secondaire à la cryoglobulinémie qui est une complication classique du syndrome de Sjögren primitif. Le pronostic rénal des patients ayant une néphrite tubulo-interstitielle est en général favorable mais le risque d'insuffisance rénale chronique reste élevé. Il est donc important que la néphrite tubulo-interstitielle soit dépistée tous les ans chez les patients qui ont un syndrome de Sjögren primitif avec atteinte systémique. Nous abordons ici l'épidémiologie, la physiopathologie, les diagnostics différentiels et le traitement des atteintes rénales spécifiques au cours du syndrome de Sjögren primitif.

\section{Mots-clés}

Syndrome de Sjögren primitif; Néphrite tubulo-interstitielle; Glomérulonéphrite membranoproliférative; Cryoglobulinémie; Acidose tubulaire distale. 
Abstract: Primary Sjögren syndrome (pSS) is an autoimmune disorder characterized by lymphoplasmacytic infiltration of the exocrine (salivary and lachrymal) glands resulting in sicca symptoms (dryness). Systemic complications can occur in pSS, but renal involvement is rare, affecting $<10 \%$ patients. The most frequent form of nephropathy in pSS is tubulointerstitial nephritis (TIN), where infiltration of the kidney by plasma cells is a key feature and shows similarity to the lymphoplasmacytic infiltration of the salivary glands. Electrolyte disturbances may occur in pSS, such as renal distal tubular acidosis, diabetes insipidus, Gitelman syndrome, or Fanconi syndrome. Glomerular involvement is less frequently detected in patients with pSS, but can take the form of membranoproliferative glomerulonephritis secondary to cryoglobulinaemia. The renal prognosis in patients with pSS and TIN or glomerular disease is usually good, but the risk of chronic kidney disease remains significant for some patients. Appropriate screening must be performed at least once a year in patients with systemic pSS in order to facilitate the early detection of renal complications. In this Review, we discuss the epidemiology, pathophysiology, differential diagnosis, and treatment of renal disease in pSS.

Keywords: primary Sjögren syndrome, tubulo-interstitial nephritis, membrano-proliferative glomerulonephritis, cryoglobulinemia, distal tubular acidosis.

\section{Conflict of interest statement:}

Helene François and Xavier Mariette have no conflict of interest relative to this publication. 


\section{Introduction}

Le syndrome de Sjögren primitif est une maladie auto-immune qui affecte 0,01 à 0,1\% de la population avec un âge moyen d'environ 50 ans, touchant plus particulièrement les femmes avec un ratio de 9/1 [1-3]]. La caractéristique principale du syndrome de Sjögren primitif est l'atteinte exocrine en raison de l'infiltration lymphoplasmocytaire qui entraîne le syndrome sec buccal et oculaire. Bien que bénins, ces symptômes, de même que la fatigue et les douleurs, peuvent affecter profondément la qualité de vie des patients au cours du syndrome de Sjögren primitif. En outre, les atteintes systémiques sont possibles et peuvent menacer le pronostic vital bien qu'elles ne concernent qu'une faible proportion de patients. La néphropathie tubulo-interstitielle est la manifestation rénale la plus fréquente et correspond à ce qui est observé au sein des glandes exocrines avec une infiltration tubulaire et interstitielle par des lymphocytes et des plasmocytes ${ }^{[[4]]}$. L'activation polyclonale B est une autre caractéristique du syndrome de Sjögren primitif et peut conduire au développement d'une cryoglobulinémie qui peut entraîner une vascularite et une glomérulonéphrite membranoproliférative, la seconde néphropathie la plus fréquente au cours du syndrome de Sjögren primitif. Du fait de l'activation chronique des lymphocytes B autoréactifs, les lymphomes B non hodgkiniens représentent la complication la plus sévère des patients atteints de syndrome de Sjögren primitif et surviennent entre 5 et $10 \%$ des cas ${ }^{[2]]}$. Les lymphomes de type mucosa-associated lymphoid tissue lymphoma (lymphomes MALT) sont la forme la plus fréquente qui affecte classiquement les glandes salivaires, les ganglions et également bien que très rarement les reins ${ }^{[5,6]]}$. Il est très important, dans cet article, de ne se focaliser que sur les atteintes rénales du syndrome de Sjögren primitif et non des syndromes de Sjögren associés à d'autres maladies auto-immunes comme le lupus qui doivent avoir été exclues. Au cours du syndrome de Sjögren primitif, l'atteinte rénale est rare d'après les plus grandes études de cohortes mais certaines séries notamment en Chine retrouvent une prévalence de près de $30 \%$ d'atteinte rénale au cours de cette maladie [4, 7-9]]. Dans cet article, seules les études traitant du syndrome de Sjögren primitif avec des critères diagnostiques solides sont considérées et nous décrivons les aspects cliniques, physiopathologiques, histologiques, les traitements et le pronostic des patients ayant une atteinte rénale spécifique au cours cette maladie.

\section{Présentation et diagnostic du syndrome de Sjögren primitif}

Un diagnostic précis et reproductible est difficile et repose sur une association de critères clinicobiologiques ainsi que de critères d'exclusion comme l'hépatite $\mathrm{C}$ chronique, le syndrome de l'immunodéficience acquise (sida), la radiothérapie cervicale et la sarcoïdose qui peuvent donner de façon non spécifique un syndrome sec ${ }^{[10]]}$. Les critères diagnostiques ont beaucoup évolué au cours des 20 dernières années commençant par un premier consensus européen en 1993 révisé en $1996^{[[11,12]]}$. Ces critères ont été revus en 2002 par un consensus américain et européen (AmericanEuropean Consensus Criteria [AECG]) et représentent les critères les plus fréquemment utilisés dans les publications bien que d'autres aient été développés par la suite en $2012^{[[10,13]]}$ puis de façon 
consensuelles en 2016 par l'American College of Rheumatology (ACR)/European League Against Rheumatism (EULAR) [14]] (Tableau 1A). Les patients atteints de syndrome de Sjögren primitif peuvent aussi développer des manifestations extraglandulaires comme des arthralgies, des atteintes pulmonaires, des neuropathies périphériques qui peuvent être difficiles à caractériser. L'EULAR a donc validé un score clinicobiologique connu sous le nom d'EULAR Sjögren's Syndrome Disease Activity Index (ESSDAI) qui permet de caractériser au mieux l'activité systémique ${ }^{[15]]}$. En utilisant ce score, les arthralgies sont la complication extraglandulaire la plus fréquente, retrouvée dans environ $30-50 \%$ des patients ${ }^{[2,8,15]]}$. Les symptômes généraux affectent $10-15 \%$ des patients avec syndrome de Sjögren primitif ; tandis que les symptômes pulmonaires, cutanés et nerveux touchent $5-10 \%$ des patients ${ }^{[[16]]}$. D'autres organes comme le rein, les ganglions, les muscles et le système nerveux central peuvent être atteints mais plus rarement ${ }^{[[1,2,7]]}$. La présence de marqueurs biologiques d'activation monoclonale B comme la gammapathie monoclonale, l'hypocomplémentémie et la cryoglobulinémie est corrélée à l'atteinte systémique ${ }^{[15]]}$ et au pronostic ${ }^{[2]]}$.

\section{Dépistage biologique recommandé au cours du syndrome de Sjögren primitif}

- Une fois par an en cas de syndrome de Sjögren primitif avec atteinte systémique

- Dosages plasmatiques : créatinine, potassium, bicarbonate, chlore

- Dosages urinaires : bandelette urinaire le matin avec $\mathrm{pH}$, osmolarité urinaire, glycosurie ; dosage du rapport protéines/créatinine sur échantillon urinaire le matin

- Tous les 6 mois si des anomalies sont détectées

- Dosages plasmatiques: créatinine, potassium, bicarbonate, chlore, phosphate, acide urique

- Dosages urinaires : bandelette urinaire le matin avec $\mathrm{pH}$, osmolarité urinaire, glycosurie ; volume urinaire des $24 \mathrm{~h}$, protéinurie, créatininurie, calciurie, citraturie, examen cytobactériologique des urines (ECBU)

- Échographie rénale

\section{- Discuter la biopsie rénale}

\section{Physiopathologie du syndrome de Sjögren primitif}

Certains facteurs environnementaux comme les infections virales ou bactériennes stimulent l'immunité innée chez les patients ayant une susceptibilité génétique ${ }^{[[17]]}$ (Fig. 1). Par ailleurs, on note la production d'interféron et d'autres cytokines participant à l'activation polyclonale des lymphocytes B qui entraîne la production des $\gamma$-globulines et autoanticorps, l'activation 
monoclonale $\mathrm{B}$, puis les lymphomes [18]]. L'épithélium glandulaire est décrit à la fois comme le déclencheur et la cible du syndrome de Sjögren primitif. Une fois activé par des virus (agonistes des TLR3 ou TLR7) ou un stress physique, les cellules épithéliales présentent des autoantigènes aux cellules dendritiques en exprimant des molécules human leukocyte antigen (HLA) de classe II (DR) activant ainsi les lymphocytes T, puis B. En outre, les cellules épithéliales sont ensuite les premières cibles au cours du syndrome de Sjögren primitif [18]]. De façon intéressante, les cellules épithéliales rénales subissent le même processus que celles des glandes salivaires puisque l'infiltrat lymphoïde est attiré au contact de l'épithélium rénal qui exprime aussi HLA DR au cours des maladies rénales [[19,20]].

\section{Prévalence de l'atteinte rénale au cours du syndrome de Sjögren primitif}

L'atteinte rénale est rare et hétérogène au cours du syndrome de Sjögren primitif et va des perturbations hydroélectrolytiques isolées avec ou sans lithiase aux glomérulopathies et néphropathie tubulo-interstitiellese développant sur le mode aigu ou chronique. En raison des changements dans les critères diagnostiques ces 20 dernières années, il reste difficile d'en établir une prévalence exacte. Par ailleurs, les syndromes de Sjögren primitif et secondaires ont souvent été associés dans diverses séries faussant ainsi sa prévalence exacte ${ }^{[21,22]]}$. Nous ne parlons ici que des séries les plus récentes postérieures aux critères AECG. La prévalence de l'atteinte rénale au cours du syndrome de Sjögren primitif varie donc d'environ $1 \%$ dans des registres rétrospectifs, à 5-14\% dans la plupart des séries européennes et supérieure à $30 \%$ dans une cohorte de 573 patients chinois (Tableau 2) [77,9,15, 23,24]]. Cette variation dans la prévalence ne peut en effet pas uniquement être due aux différents critères diagnostiques utilisés dans ces études. Par exemple, les manifestations tubulaires, bien que fréquentes, ne sont pas toujours retrouvées à moins d'être recherchées de façon plus systématique. Leur fréquence pourrait s'en trouver sous-estimée [[4]]. Au cours de l'une de ces études où les fonctions tubulaires avaient été systématiquement testées par une recherche de troubles de la concentration urinaire et d'acidose tubulaire, il a été retrouvé que $27 \%$ des patients avaient une atteinte tubulaire au cours du syndrome de Sjögren primitif. Bien que des tests fonctionnels urinaires soient compliqués à réaliser en pratique courante, un simple dosage des bicarbonates sanguins, de la kaliémie, ainsi qu'une mesure du $\mathrm{pH}$ urinaire et de l'osmolarité urinaire sur échantillon à jeun sont des tests faciles à réaliser avec une simple bandelette. De façon intéressante, de tels tests n'ont pas été réalisés dans la cohorte chinoise [99]]. Toutefois, 44 des 573 patients ont par la suite développé d'autres maladies autoimmunes, incluant le lupus moins de trois ans après le diagnostic de syndrome de Sjögren primitif. Bien que les critères AECG aient été utilisés, la nature primitive du syndrome de Sjögren dans cette cohorte est donc sujette à caution.

\section{Présentation de l'atteinte rénale au cours du syndrome de Sjögren primitif}

Beaucoup d'atteintes différentes sont associées au syndrome de Sjögren primitif et les plus fréquentes sont représentées par la néphropathie tubulo-interstitielleet la glomérulonéphrite 
membranoproliférative associée à la cryoglobulinémie (Tableau 3). Les symptômes rénaux surviennent en général chez des patients d'âge supérieur ou égal à 50 ans, même si les patients de la cohorte chinoise avaient moins de 40 ans en moyenne ${ }^{[8,24,25]]}$. Le syndrome de Sjögren primitif est très rare chez les enfants et adolescents et l'atteinte rénale est peu souvent décrite dans cette population ${ }^{[23,26,27]]}$. Dans la plupart des séries, les symptômes rénaux sont en général retrouvés entre 2-7 ans après le diagnostic initial de syndrome de Sjögren primitif ${ }^{[4,24,25]]}$. Toutefois, une étude a retrouvé que la néphropathie tubulo-interstitiellesurvenait en moyenne plus tôt ( 2 ans) après le diagnostic de syndrome de Sjögren primitif que la glomérulonéphrite membranoproliférative secondaire à la cryoglobulinémie (8 ans) ${ }^{[24]]}$; mais cela n'a pas été confirmé par une autre étude, qui retrouvait que les symptômes rénaux survenaient en moyenne 2 à 3 ans après le diagnostic de syndrome de Sjögren primitif quelle que soit l'histologie [25]]. En revanche, dans la série de biopsies rénales la plus importante d'Europe (95 cas de patients avec syndrome de Sjögren primitif biopsies), l'atteinte rénale était concomitante du diagnostic dans plus de $50 \%$ des cas [[28]]. Les atteintes rénales font partie du score ESSDAI ${ }^{[15]]}$ qui permet de définir l'activité du syndrome de Sjögren primitif. La classification de l'atteinte rénale va de l'absence d'atteinte spécifique (sédiment urinaire normal, protéinurie inférieure à $0,5 \mathrm{~g} / 24 \mathrm{~h}$, pas d'acidose tubulaire et fonction rénale stable) à une atteinte très active (définie par une atteinte glomérulaire proliférative avec protéinurie de fort débit, hématurie et insuffisance rénale chronique avec un débit de filtration glomérulaire inférieur à $60 \mathrm{ml} / \mathrm{min} / 1,73 \mathrm{~m}^{2}$ ). Les maladies glomérulaires actives sont définies par le degré de la protéinurie, la présence d'une hématurie, qui en général correspond au degré de prolifération glomérulaire et donc à sa sévérité. Le score ESSDAI est très utile pour décrire l'activité de la maladie rénale mais ne décrit pas de façon précise la néphropathie tubulo-interstitielle. En effet, en général la néphropathie tubulo-interstitielle est associée à une faible activité de la maladie puisque la fonction rénale se détériore en général lentement contrairement à la glomérulonéphrite membranoproliférative. Cependant, l'insuffisance rénale terminale reste possible chez les patients atteints de néphropathie tubulo-interstitielle au cours du syndrome de Sjögren primitif et un suivi est bien sûr nécessaire également chez ces patients. Les différentes présentations cliniques possibles sur le plan rénal sont résumées dans le Tableau 3.

\section{Néphropathie tubulo-interstitielle}

\subsection{Présentation clinique, pronostic et traitement}

La néphropathie tubulo-interstitielle a été décrite approximativement chez deux tiers des patients atteints de syndrome de Sjögren primitif ayant eu une biopsie rénale ${ }^{[4,23-25,28]]}$ (Tableau 4). La maladie est habituellement insidieuse avec peu de symptômes cliniques autres que des désordres hydroélectrolytiques, une élévation de la créatininémie et une protéinurie de faible débit. La survenue de la néphropathie tubulo-interstitielle ne semble pas affecter le pronostic vital des patients [24]]. L'identification de la protéinurie peut être difficile puisque la protéinurie tubulaire n'est pas détectée 
par les bandelettes urinaires habituellement utilisées en pratique clinique pour le dépistage des maladies rénales, qui ne détectent en effet que l'albumine. En outre, l'hypertension artérielle n'est présente qu'aux stades tardifs d'insuffisance rénale chronique (4 ou 5) au cours des néphropathies tubulo-interstitielles. Ainsi, sans dépistage spécifique, les néphropathies tubulo-interstitielles ne sont souvent pas diagnostiquées, ce qui explique sans doute la faible prévalence des atteintes rénales dans

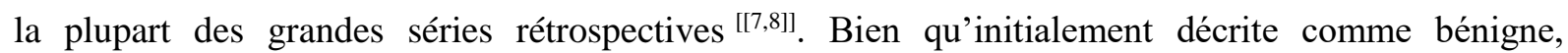
l'insuffisance rénale terminale est possible ${ }^{[[23,24,28,29]]}$. C'est pourquoi une recherche spécifique de la néphropathie tubulo-interstitielle doit être réalisée au moins une fois par an chez les patients avec syndrome de Sjögren primitif et complications systémiques avec une recherche de protéinurie (soit des $24 \mathrm{~h}$, soit sur échantillon, couplée au dosage de la créatininurie), créatininémie, débit de filtration glomérulaire estimé et ionogramme sanguin. L'atteinte rénale est souvent difficile à établir cliniquement, c'est pourquoi la biopsie rénale doit être pratiquée rapidement en cas de doute.

La plupart des patients développent une insuffisance rénale aiguë de sévérité modérée mais elle est le plus souvent subaiguë ou chronique avec une protéinurie tubulaire (faite de protéines de bas poids moléculaire) et acidose tubulaire distale ${ }^{[23]]}$. Bien que 1' acidose tubulaire distale soit la plus fréquente des acidoses tubulaires rénales au cours du syndrome de Sjögren primitif, le syndrome de Fanconi (touchant les tubes proximaux) a également été décrit ${ }^{[4,23,25]]}$. L'inflammation est souvent chronique au cours de la néphropathie tubulo-interstitielle et elle entraîne une fibrose interstitielle et une insuffisance rénale chronique dans les formes persistantes. Le diagnostic étant parfois tardif et la réponse au traitement parfois médiocre, fibrose interstitielle et atrophie tubulaire peuvent donc se développer de façon chronique et conduire à l'insuffisance rénale terminale ${ }^{[24,25]]}$. Dans la plupart des séries, la réponse rénale à l'utilisation de corticoïdes seuls à des doses supérieures à $0,5 \mathrm{mg} / \mathrm{kg} / \mathrm{j}$ était bonne avec amélioration de la fonction rénale ${ }^{[23]]}$. La normalisation complète de la protéinurie et de la fonction rénale n'est souvent pas atteinte au cours des néphropathies tubulo-interstitielles, possiblement en raison de lésions de fibrose interstitielle ${ }^{[[4,23]]}$.

Il n'existe pas de facteurs pronostiques clairement identifiés pour le développement de la néphropathie tubulo-interstitielle dans la majorité des séries ${ }^{[24]]}$ sauf la présence concomitante d'anticorps anti-SSA (Ro) et SSB (La) qui serait péjorative dans une étude récente [28]]. L'autre question non résolue concerne le traitement: l'adjonction d'immunosuppresseurs permet-elle un meilleur contrôle de l'inflammation tubulo-interstitielle que les stéroïdes seuls ? Jusqu'à présent, y compris dans l'étude la plus récente de la plus grande série européenne, aucune publication ne prouve la supériorité de l'ajout d'un immunosuppresseur sur le déclin de la fonction rénale (cyclophosphamide, azathioprine, mycophénolate mofétil ou rituximab compris) en plus des stéroïdes au cours de l'atteinte rénale du syndrome de Sjögren primitif [28]]. Comme dans toutes les maladies auto-immunes, l'espoir est que l'adjonction d'un traitement immunosuppresseur permette une épargne cortisonique. Le choix de l'immunosuppresseur (rituximab, mycophénolate mofétil, ou azathioprine) pourrait être guidé par la composition de l'infiltrat cellulaire interstitiel rénal, bien qu'aucune donnée 
actuelle ne le prouve. Étant donné la toxicité du cyclophosphamide, il ne semble pas raisonnable d'utiliser cette thérapie en première ligne. De même, les anomalies hydroélectrolytiques isolées avec une fonction rénale stable et normale peuvent n'être traitées que par supplémentation en bicarbonate ou citrate de potassium selon les troubles lorsqu'ils ne sont pas associés à une néphropathie tubulointerstitielle. Toutefois, dans la majorité des cas, les désordres hydroélectrolytiques sont la conséquence de la néphropathie tubulo-interstitielle avec lésions de tubulites qui répondent le plus souvent aux stéroïdes comme discuté précédemment ${ }^{[23,30]]}$.

\subsection{Physiopathologie}

La dysfonction rénale peut être la conséquence de l'infiltration tubulo-interstitielle par les lymphocytes T, B et des plasmocytes et plus rarement par des autoanticorps [[31,32]]. La plupart des symptômes observés sont une conséquence de l'infiltration lymphocytaire qui entraîne par la suite la fibrose interstitielle et l'insuffisance rénale chronique (Fig. 1). Les lésions de tubulites sont aussi associées à l' acidose tubulaire distale et conduisent à l'absence complète de pompe $\mathrm{H}^{+}$-ATPase dans les canaux collecteurs et de $\mathrm{NaCl}$ cotransporteur thiazide-sensible (NCCT) ${ }^{[[1,32,33]]}$. Des autoanticorps ont été retrouvés contre le $\mathrm{NaCl}$ cotransporteur thiazide-sensible, bien que leur rôle dans le

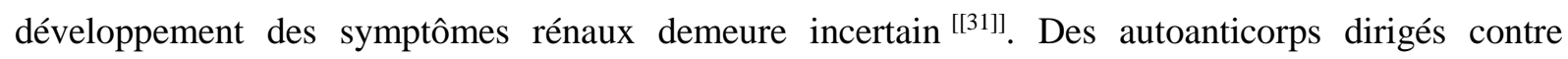
l'anhydrase carbonique peuvent être retrouvés dans le sérum des patients souffrant de lupus et de syndrome de Sjögren primitif et ont également été détectés dans les tubes distaux [[34]]. En outre, les autoanticorps contre l'anhydrase carbonique ont été identifiés chez des patients atteints de syndrome de Sjögren primitif et semblent corrélés à la présence de 1 ' acidose tubulaire distale ${ }^{[[35]]}$. Il reste cependant à définir si ces autoanticorps sont la conséquence ou contribuent aux lésions rénales bien que des anticorps dirigés contre l'anhydrase carbonique chez la souris aient pu reproduire l' acidose tubulaire distale ${ }^{[[36]]}$. Les cibles antigéniques de certains de ces anticorps (par exemple, l'anhydrase carbonique II et la $\mathrm{H}^{+}$-ATPase) sont retrouvées aussi bien dans les glandes salivaires que dans les cellules intercalaires de type A rénales ${ }^{[37]]}$. Les lésions des glandes salivaires peuvent donc être la cause du relargage d'antigènes dans la circulation sanguine, puis le rein et deviennent ensuite la cible des lymphocytes $\mathrm{T}$ et des autoanticorps. En outre, certaines études ont retrouvé une corrélation positive entre la durée de la maladie, l'hypergammaglobulinémie et la présence d'autoanticorps contre SSA (Ro) et SSB (La) ${ }^{[[4,22,28,38,39]]}$.

Les anomalies tubulaires et notamment sur les cellules intercalaires de type A conduisent à un défaut d'excrétion de la charge acide (donc un défaut d'acidification urinaire) qui peut conduire à une acidose métabolique hyperchlorémique. Cette acidose entraîne une résorption osseuse accrue et donc une hypercalciurie favorisant les lithiases oxalocalciques. Une hypocitraturie peut également être présente au cours de l' acidose tubulaire distale par augmentation de sa réabsorption tubulaire proximale qui favorise également la formation lithiasique. Dans certains cas, les dépôts oxalocalciques 
tubulaires conduisent à une néphrocalcinose au cours des hypercalciuries chroniques, même si cette pathologie demeure très rare au cours du syndrome de Sjögren primitif.

\subsection{Histologie rénale}

La plupart du temps les lymphocytes $\mathrm{T}$ et $\mathrm{B}$ infiltrent le rein en proportion équivalente au cours de la néphropathie tubulo-interstitielle et l'infiltrat peut être focal ou diffus et les cellules B ne prédominent que dans $10 \%$ des cas. L'infiltration plasmocytaire est fréquente au cours de la néphropathie tubulo-interstitielle survenant dans plus de $70 \%$ des cas ${ }^{[4,28]]}$ et les plasmocytes peuvent même être la cellule prédominant dans l'infiltrat cellulaire dans près de $25 \%$ des cas [28]]. L'histologie de la néphropathie tubulo-interstitielle n'est pas spécifique sauf cette infiltration fréquente par des cellules B et des plasmocytes (Fig. 2, 3) . La néphropathie tubulo-interstitielle pourrait donc survenir au cours des syndrome de Sjögren primitif les plus sévères avec signes d'activation chronique B. C'est pourquoi, l'utilisation d'immunosuppresseurs ciblant la cellule B comme le rituximab ou les inhibiteurs du protéasome pourraient être des pistes thérapeutiques prometteuses. L'inflammation locale déclenche les lésions tubulaires qui conduisent à leur atrophie et à la fibrose interstitielle et ainsi à l'insuffisance rénale chronique.

\subsection{Diagnostics différentiels}

En plus de suivre les critères diagnostiques du syndrome de Sjögren primitif, une recherche soigneuse de signes extrarénaux doit être réalisée chez les patients atteints de néphropathie tubulointerstitielle . Cette étape est importante puisque aucune lésion rénale spécifique n'a jusqu'alors été décrite et que le diagnostic du syndrome de Sjögren primitif est très souvent concomitant du diagnostic de l'atteinte rénale ${ }^{[[28,33]]}$.

\subsubsection{Maladie associée aux immunoglobulines $G_{4}\left(I g G_{4}\right)$}

Bien que les cellules plasmocytaires soient fréquemment retrouvées au cours de la néphropathie tubulo-interstitielle du syndrome de Sjögren primitif, elles ne sont pas spécifiques et sont également l'un des signes principaux au cours de la maladie des $\operatorname{IgG}_{4}{ }^{[400]]}$. Toutefois, la fibrose dite storiformelike est souvent caractéristique de la maladie et l'infiltrat plasmocytaire prend souvent un aspect pseudotumoral ${ }^{[[40]]}$. Les critères diagnostiques incluent un ratio plasmocyte $\mathrm{IgG}_{4}+/ \operatorname{IgG}+$ supérieur à $40 \%$ ou supérieur ou égal à dix $\mathrm{IgG}_{4}+$ plasmocytes par champ ${ }^{[40]]}$. La maladie associée aux $\mathrm{IgG}_{4}$ partage beaucoup de caractéristiques cliniques avec le syndrome de Sjögren primitif dont l'hypergammaglobulinémie, l'infiltration des glandes salivaires et l'hypocomplémentémie. Une élévation des $\mathrm{IgG}_{4}$ est également retrouvée chez environ $7 \%$ des patients avec syndrome de Sjögren primitif ${ }^{[[41]]}$ et des plasmocytes $\operatorname{IgG}_{4}+$ ont déjà été détectés dans des biopsies de glandes salivaires ou rénales au cours du syndrome de Sjögren primitif ${ }^{[[42,43]]}$, ce qui peut rendre la distinction entre les deux maladies difficile. Cependant, la maladie associée aux $\operatorname{IgG}_{4}$ affecte principalement les hommes âgés de plus de 60 ans, ne s'accompagne pas d'anticorps anti-SSA et anti-SSB et l'organisation 
pseudotumorale de l'infiltrat n'est pas classiquement retrouvée au cours du syndrome de Sjögren primitif. Des critères diagnostiques solides peuvent donc aider à la différenciation de ces deux maladies.

\subsubsection{Sarcö̈dose}

La sarcoïdose est une autre cause classique de néphropathie tubulo-interstitielle tout comme le syndrome tubulo-interstitial nephritis and uveitis (TINU) et les néphropathies tubulo-interstitielles idiopathiques [[44]]. Les granulomes rénaux ne sont classiquement pas décrits au cours des néphropathies tubulo-interstitielles du syndrome de Sjögren primitif mais ont pu être retrouvés au niveau des glandes salivaires. Ainsi le diagnostic différentiel peut être difficile et l'association des deux maladies a été décrite ${ }^{[23,45]]}$.

\section{Troubles hydroélectrolytiques}

En dehors de la véritable néphropathie tubulo-interstitielle symptomatique, les désordres hydroélectrolytiques peuvent être le premier symptôme de la maladie rénale au cours du syndrome de Sjögren primitif et apparaissent souvent de façon isolée. Toutefois dans la majorité des cas, les désordres hydroélectrolytiques sont associés à la néphropathie tubulo-interstitielle ${ }^{[[4,23,24]]}$ et une biopsie rénale est recommandée à moins que d'autres causes d' acidose tubulaire distale ne soient suspectées. De façon plus spécifique, les lésions de tubulites et d'atrophie tubulaire peuvent être responsables de défauts de l'acidification urinaire et de troubles de la concentration urinaire [[23]]. Tous les segments du néphron peuvent être en cause mais les plus classiquement atteints sont les tubules distaux et les canaux collecteurs. Les diagnostics différentiels concernant les troubles hydroélectrolytiques au cours du syndrome de Sjögren primitif sont résumés dans le Tableau 5.

\subsection{Acidose tubulaire distale}

L'acidose tubulaire distale est l'anomalie hydroélectrolytique la plus fréquente au cours du syndrome de Sjögren primitif et touche les canaux connecteurs et collecteurs. De nombreuses maladies peuvent causer une acidose tubulaire distale et il est important d'en rechercher d'autres causes comme les formes familiales d'acidose tubulaire distale et le lupus quand l'hypokaliémie est présente. Dans certains cas, l'hypokaliémie peut être le seul symptôme biologique et est parfois accompagnée de crampes et d'épisodes de paralysie périodique. Un autre symptôme qui peut conduire au diagnostic de l'acidose tubulaire distale est la lithiase secondaire à l'hypercalciurie et l'hypocitraturie, voire la néphrocalcinose ${ }^{[[4,23,24]]}$. Si le potassium est normal ou élevé, le syndrome de Sjögren primitif n'est en général pas en cause. L' acidose tubulaire distale complète est caractérisée par une acidose métabolique avec un pH urinaire supérieur à 5,5 et un trou anionique urinaire positif. Les acidoses tubulaires distale incomplètes se présentent avec un taux plasmatique de bicarbonate sanguin normal mais le $\mathrm{pH}$ urinaire ne descend pas en dessous de 5,3 après une charge acide [[46]]. L'acidose tubulaire distale incomplète ne peut donc être retrouvée qu'en cas de réalisation d'un test de charge acide. La 
prévalence de l'acidose tubulaire distale complète a été rapportée allant de moins de $5 \%$ dans une cohorte de patients italiens avec syndrome de Sjögren primitif et à $33 \%$ aux États-Unis et jusqu'à $70 \%$ en Chine ${ }^{[[4,23,25]]}$.

\subsection{Troubles de la concentration urinaire}

Les troubles de la concentration urinaire se manifestent par une nycturie, une polyurie et un diabète insipide classiquement retrouvé au cours du syndrome de Sjögren primitif et touchent également le canal collecteur ${ }^{[4,24,25]]}$. Dans la cohorte italienne, ils ont été retrouvés chez 13 patients sur 48 atteints d'un syndrome de Sjögren primitif après avoir réalisé un test de restriction hydrique ${ }^{[[4]]}$. La moitié de ces patients n'avait pas d'altération notable de la fonction rénale. Les symptômes de troubles de la concentration urinaire sont en général discrets et ne sont pas toujours décrits ou avec une prévalence très faible ${ }^{[25,29]]}$. Ils peuvent être en partie liés à l' acidose tubulaire distale si elle a pour conséquence une hypokaliémie chronique ou une néphrocalcinose (par défaut de sensibilité tubulaire à l'hormone antidiurétique).

\subsection{Acidose tubulaire proximale}

D'autres segments peuvent être touchés autres que les plus distaux bien que cela soit nettement moins fréquent. En effet, l'acidose tubulaire proximale (ou syndrome de Fanconi) est rare au cours du syndrome de Sjögren primitif et affecte uniquement environ 3-4\% des patients ${ }^{[23,25]]}$. Une acidose métabolique avec un trou anionique plasmatique normal, un phosphate bas et un acide urique plasmatique bas évoque plutôt le caractère proximal que distal de l'acidose (quand associés avec une perte urinaire). La glycosurie normoglycémique peut également être utilisée comme marqueur spécifique d'acidose tubulaire proximale bien que ces tests ne soient pas réalisés en pratique clinique courante $^{[[47]]}$. Les symptômes cliniques du syndrome de Fanconi sont très rares et comprennent le rachitisme, les fractures osseuses et l'ostéomalacie ${ }^{[[47]]}$. L'acidose tubulaire proximale correspond également à l'atrophie tubulaire au cours de la néphropathie tubulo-interstitielle du syndrome de Sjögren primitif [[30]]. De même que pour l' acidose tubulaire distale, il faut rechercher des diagnostics différentiels comme les formes héréditaires d'acidose tubulaire proximale (cystinose et tyrosinémie qui sont plutôt rencontrées chez les enfants). Il existe surtout chez l'adulte des formes acquises de syndrome de Fanconi secondaires aux gammapathies monoclonales, à certains médicaments (ifosfamide ou ténofovir) ou à des toxiques (plomb notamment).

\subsection{Syndrome de Gitelman acquis}

Le syndrome de Gitelman est une maladie autosomique récessive qui est causée par une mutation de $S L C 12 A 3$ codant pour le tubule $\mathrm{NaCl}$ cotransporteur thiazide-sensible ${ }^{[[48]}$. Des syndromes de Gitelman acquis ont été décrits chez des patients atteints de néphropathie tubulo-interstitielle au cours du syndrome de Sjögren primitif [31,49]]. Le syndrome de Gitelman correspond à une hypokaliémie d'origine rénale (avec une concentration urinaire de potassium supérieure à $20 \mathrm{mmol} / \mathrm{l}$ ) et une 
hypomagnésémie associée à un hyperaldostéronisme secondaire due à une contraction du volume extracellulaire (rénine et aldostérone élevées avec alcalose métabolique de contraction). Bien que la majorité des syndromes de Gitelman acquis soient secondaires aux lésions de tubulites au cours de la néphropathie tubulo-interstitielle, une étude a rapporté un cas de syndrome de Gitelman associé à un autoanticorps dirigé contre le $\mathrm{NaCl}$ cotransporteur thiazide-sensible détecté dans le sérum du patient ${ }^{[31,49]]}$.

\subsection{Syndrome de Bartter acquis}

Le syndrome de Bartter peut se développer au cours du syndrome de Sjögren primitif mais il a été très rarement rapporté [[50]]. De façon proche du syndrome de Gitelman, le syndrome de Bartter correspond à une hypokaliémie d'origine rénale avec hyperaldostéronisme secondaire dû à la perte de sel. À l'inverse du syndrome de Gitelman, la contraction des volumes extracellulaires est en général plus prononcée et les patients présentent une hypercalciurie.

\section{Glomérulonéphrite membranoproliférative}

\subsection{Présentation clinique, pronostic et traitement}

Le syndrome de Sjögren primitif est la maladie auto-immune la plus fréquemment retrouvée au cours des cryoglobulinémies non virus de l'hépatite C (VHC) représentant environ 30-45\% des cas ${ }^{[51,52]]}$. La glomérulonéphrite membranoproliférative (de type 1 mais avec quelques spécificités histologiques) secondaire à une cryoglobulinémie survient dans 5 à $30 \%$ des patients syndrome de Sjögren primitif ayant bénéficié d'une biopsie rénale (Tableau 4) ${ }^{[23,24,28,53]]}$. La présentation rénale au cours de la glomérulonéphrite membranoproliférative est le plus souvent cliniquement très parlante avec une hypertension artérielle, une protéinurie de fort débit, hématurie et une insuffisance rénale aiguë correspondant à un syndrome néphrotique ou à une glomérulonéphrite rapidement progressive ${ }^{[51,52]]}$. La glomérulonéphrite membranoproliférative secondaire à une cryoglobulinémie est une complication mettant en jeu le pronostic vital qui impacte la survie en raison du développement de lésions de vascularite systémique. La survie de ces patients est significativement diminuée par rapport aux patients atteints de syndrome de Sjögren primitif souffrant de néphropathie tubulo-interstitielle bien que l'insuffisance rénale chronique soit moins fréquente [[24]]. Contrairement à la néphropathie tubulo-interstitielle, l'atteinte rénale au cours de la glomérulonéphrite membranoproliférative est très symptomatique et est en effet diagnostiquée souvent plus rapidement. Les traitements le plus souvent utilisés sont les stéroïdes (la plupart du temps en bolus à fortes doses), des immunosuppresseurs comme le cyclophosphamide, le rituximab, l'azathioprine et le mycophénolate mofétil en combinaison avec des échanges plasmatiques ${ }^{[23-25]]}$. La plupart des études rapportent une amélioration notable de la fonction rénale sous traitement avec peu d'insuffisance rénale chronique ${ }^{[24]]}$. De façon similaire, les études rapportant le pronostic de patients atteints de cryoglobulinémie non VHC ont retrouvé qu'une combinaison d'échanges plasmatiques et de rituximab 
est le traitement de choix avec un bon pronostic rénal [51,52]]. Le rituximab est également efficace dans les manifestations systémiques des cryoglobulinémies positives au VHC et ce traitement est largement utilisé au cours des manifestations systémiques de la cryoglobulinémie au cours du syndrome de Sjögren primitif et particulièrement pour la neuropathie périphérique bien que sa supériorité par rapport aux autres immunosuppresseurs n'ait pas été formellement démontrée [[54-57]].

\subsection{Physiopathologie}

La glomérulonéphrite membranoproliférative secondaire à la cryoglobulinémie est une conséquence directe de la stimulation constante des lymphocytes B. Cette glomérulopathie fait partie, dans la nouvelle classification des glomérulonéphrite membranoproliférative, de celles qui sont médiées par les complexes immuns ${ }^{[[58]]}$ puisque les dépôts sont constitués d'immunoglobulines (IgG, $\operatorname{IgM}, \operatorname{IgA}, \kappa$ et $\lambda)$ et de complément $(\mathrm{C} 3, \mathrm{C} 1 \mathrm{q})$, plutôt que de dépôts exclusifs de $\mathrm{C} 3$ qui sont rencontrés au cours des glomérulonéphrite membranoproliférative médiées par le complément [[58]]. L'hypergammaglobulinémie et la formation des complexes immuns sont une particularité du syndrome de Sjögren primitif. Ces complexes immuns stimulent les lymphocytes B via leur fragment Fc à activité anti-IgG (ou facteur rhumatoïde). Ce facteur rhumatoïde peut précipiter avec les

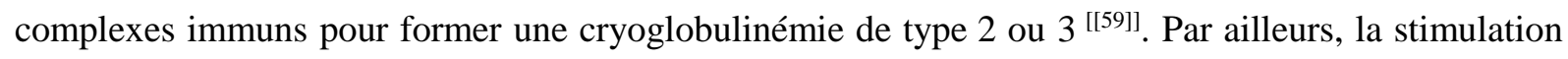
chronique des lymphocytes $\mathrm{B}$ à activité facteur rhumatoïde déclenche le développement de gammapathies monoclonales et de lymphomes [[18]]. La nature de l'autoantigène au cours du syndrome de Sjögren primitif n'est pas connue bien que la destruction des cellules épithéliales des glandes salivaires par des virus ou des lymphocytes T entraîne le relargage de nombreux autoantigènes dans la circulation qui peuvent faire partie des complexes immuns responsables de l'activation chronique des lymphocytes B et des plasmocytes. Ces complexes immuns, seuls ou associés au facteur rhumatoïde, sont déposés sous forme de cryoglobuline dans les glomérules où ils vont activer la voie classique d'activation du complément et déclenchent ainsi les lésions mésangiales et des espaces sousendothéliaux, suivie ensuite d'une phase inflammatoire, puis de réparation avec fibrose ${ }^{[58]]}$. Pendant cette phase finale, la prolifération des cellules mésangiales est accompagnée de synthèse de protéines de la matrice extracellulaire qui conduit à la formation d'un épaississement de la membrane basale glomérulaire avec doubles contours, qui est l'une des spécificités des glomérulonéphrite membranoproliférative ${ }^{[[33]]}$. En outre, la précipitation intravasculaire de cryoglobuline forme des thrombi, ce qui est également caractéristique de la glomérulonéphrite membranoproliférative au cours du syndrome de Sjögren primitif [53]]. Parfois, seule une glomérulonéphrite proliférative résultant de ces complexes immuns est retrouvée même en l'absence de cryoglobulinémie [25]].

\subsection{Histologie rénale}

Les lésions glomérulaires sont rares au cours du syndrome de Sjögren primitif et beaucoup de néphropathies glomérulaires s'accompagnent d'un infiltrat tubulo-interstitiel significatif ${ }^{[24,25,28]]}$, de 
façon similaire à ce qui est observé au cours du lupus [[60]]. La glomérulonéphrite membranoproliférative secondaire à la cryoglobuline a les mêmes caractéristiques histologiques que celle non associée aux cryoglobulinémies : c'est-à-dire une prolifération et une expansion mésangiales, un épaississement de la membrane basale glomérulaire et la formation de doubles contours (Fig. 4, 5). L'immunofluorescence est nécessaire en revanche pour faire le diagnostic afin de détecter la localisation intravasculaire et la nature des dépôts. La plupart des dépôts sont donc retrouvés de façon majoritaire dans le mésangium et les espaces sous-endothéliaux et également de façon tout à fait caractéristique aux glomérulonéphrite membranoproliférative secondaires aux cryoglobulinémies dans le secteur intravasculaire et correspondent alors au cryoprécipité [[53]]. Ils sont composés d'IgG et d'IgM. L'autre particularité de ce cryoprécipité réside également dans l'ultrastructure microtubulaire qui peut se voir en microscopie électronique. Les autres caractéristiques des glomérulonéphrite membranoproliférative secondaires aux cryoglobulinémies incluent une lobulation du flocculus en raison de la prolifération mésangiale, l'infiltration du flocculus par des monocytes et des lymphocytes $\mathrm{T}$ et l'infiltration périvasculaire par des monocytes et des lymphocytes $\mathrm{T} \mathrm{CD}^{+[[53]]}$. La prolifération extracapillaire n'est pas fréquente mais des lésions de vascularite des petits et moyens vaisseaux sont possibles de même que des thrombi intravasculaires liés au cryoprécipité.

\subsection{Diagnostic différentiel}

Le lupus érythémateux disséminé et les autres causes de cryoglobulinémie sont les diagnostics différentiels de la glomérulonéphrite membranoproliférative médiée par les complexes immuns [[58]] (Tableau 5). Les autres diagnostics différentiels des glomérulonéphrite membranoproliférative avec cryoglobulinémie incluent les infections chroniques (endocardite et infection de shunt atrioventriculaire), les infections virales chroniques (hépatites B et C) et les gammapathies monoclonales. En effet, excepté l'infiltrat tubulo-interstitiel lymphoplasmocytaire retrouvé fréquemment au cours des atteintes glomérulaires du syndrome de Sjögren primitif [28]], il n'existe aucune différence histologique avec ces autres causes de glomérulonéphrite membranoproliférative liées aux cryoglobulinémie.

\section{Néphropathies glomérulaires non spécifiques}

\subsection{Glomérulonéphrite extramembraneuse}

Le syndrome de Sjögren primitif a été longtemps classiquement décrit comment pouvant être responsable de glomérulonéphrite extramembraneuse secondaire, mais de très rares cas ont été décrits ${ }^{[61-63]]}$. La glomérulonéphrite extramembraneuse n'est retrouvée que dans moins de $10 \%$ des patients avec syndrome de Sjögren primitif ayant subi une biopsie rénale ${ }^{[[4,23-25,28]]}$ (Tableau 4). Si l'association entre le lupus et la glomérulonéphrite extramembraneuse est très robuste, celle avec le syndrome de Sjögren primitif est beaucoup plus difficile à démontrer, ce d'autant que la glomérulonéphrite extramembraneuse est fréquente chez les adultes. Jusqu'à présent, il n'a jamais été 
décrit d'autoantigène podocytaire au cours du syndrome de Sjögren primitif bien que des complexes immuns pourraient être formés de façon non spécifique en situation extramembraneuse ${ }^{[[64]]}$. Au total, il n'y a aucune preuve d'une association possible entre les deux.

\subsection{Autres néphropathies glomérulaires}

D'autres néphropathies glomérulaires ont été décrites en association avec le syndrome de Sjögren primitif comme la néphropathie à IgA (1-22\%), la hyalinose segmentaire et focale (1-8\%) et les lésions glomérulaires minimes (2-4\%) (Tableau 4). Cependant, dans la plupart des cas associant des lésions de hyalinose segmentaire et focale au syndrome de Sjögren primitif, la protéinurie était souvent de débit non néphrotique, ce qui remet en cause cette association. Des glomérulonéphrites prolifératives sans caractéristiques précises ont également été identifiées, ce qui représente sans doute une forme mineure de glomérulonéphrite membranoproliférative avec une prolifération mésangiale mais sans l'organisation lobulaire ou les doubles contours ${ }^{[9,24]]}$. Différents cas et une petite série de sept patients ont décrit l'association entre les vascularites à anticorps anticytoplasme des polynucléaires neutrophiles (ANCA) et le syndrome de Sjögren primitif [[65-69]]. Les ANCA sont retrouvés dans 6-17 \% des patients avec syndrome de Sjögren primitif et la majorité de ces patients ne développe pas de lésions de vascularite ${ }^{[[69]]}$. Bien que ces glomérulonéphrites soient d'origine autoimmune, il n'y a toujours pas à ce jour de preuve pour une association spécifique avec le syndrome de Sjögren primitif.

\section{Lymphome rénal}

Bien que le lymphome survienne dans près de $10 \%$ des patients avec syndrome de Sjögren primitif, il existe très peu de cas rapportés de lymphomes rénaux ${ }^{[5,6]]}$. Les patients ont alors une masse rénale et le diagnostic est confirmé par la biopsie qui retrouve une infiltration monotypique de lymphocytes, alors qu'ils sont polyclonaux au cours de la néphropathie tubulo-interstitielle.

\section{Recommandations de prise en charge de l'atteinte rénale au cours du syndrome de}

\section{Sjögren primitif}

Aucune étude randomisée prospective n'a précisément étudié les traitements au cours des atteintes rénales du syndrome de Sjögren primitif. Cependant, d'après les diverses séries rétrospectives publiées et récemment revues ${ }^{[[44]] 1}$, il semble raisonnable de proposer les schémas thérapeutiques qui paraissent le mieux documentés.

- Pour les troubles hydroélectrolytiques strictement isolés (c'est-à-dire avec une fonction rénale normale et parfaitement stable), la supplémentation simple est possible (potassium, citrate de potassium) afin de lutter contre l'hypokaliémie et le risque de lithiase induit par l'hypercalciurie. La biopsie doit être faite afin d'exclure ou confirmer une néphropathie tubulo-interstitielle en cas de dégradation de la fonction rénale. 
- En cas de néphropathie tubulo-interstitielle, le traitement de première intention demeure la corticothérapie à des doses supérieures à $0,5 \mathrm{mg} / \mathrm{kg}$ pour plusieurs semaines. Les thérapies ciblant le lymphocyte B peuvent être données en cas de forme réfractaire ou de cortico-dépendance (et ce d'autant plus volontiers que l'infiltrat interstitiel est majoritairement constitué de lymphocytes B et plasmocytes) ${ }^{[28]]}$. Ce traitement semble efficace même s'il n'y a pas de preuve de sa supériorité ou non-infériorité par rapport à la corticothérapie [28,57]]. La place d'autres immunosuppresseurs (azathioprine, mycophénolate mofétil) est mal codifiée et doit être réservée aux formes réfractaires ou en cas d'intolérance à la corticothérapie en alternative ou en cas d'échec du rituximab. Une série rétrospective récente documente l'efficacité sur la fonction rénale d'une association de stéroïdes avec sevrage en 3 à 6 mois et de mycophénolate mofétyl pour une durée de 24 mois ${ }^{70}$ Le pronostic rénal semble globalement bon sous traitement avec une progression très lente de l'insuffisance rénale chronique sous traitement ${ }^{[23,28]]}$. Les néphropathies tubulo-interstitielles, surtout celles ne bénéficiant pas de traitement, peuvent évoluer vers l'insuffisance rénale terminale même si l'évolution reste lente [24,44]]71-72. Il est intéressant de noter que certains patients non traités avec néphrite tubulo-interstitielle ne semblent pas dégrader leur fonction rénale ${ }^{71-28}$. De même, la corticothérapie ne semble pas toujours efficace à corriger les troubles hydroélectrolytiques ${ }^{1}$.

- En cas de glomérulonéphrite membranoproliférative avec cryoglobulinémie, le pronostic rénal semble meilleur (sans doute lié au diagnostic plus précoce) ${ }^{[28]]}$. Le traitement de choix reste la corticothérapie associée au rituximab et dans certains cas aux échanges plasmatiques. Le cyclophosphamide est également prescrit en cas de prolifération extracapillaire rénale et de vascularite cryoglobulinémique. Le rituximab améliore nettement le pronostic rénal mais semble associé à une morbidité plus importante, surtout en cas de corticothérapie forte et prolongée [[52]]. Une étude prospective randomisée est en cours afin d'évaluer la place du rituximab dans cette indication même si les données rétrospectives semblent très solides [[44]].

\section{Conclusion}

Le pronostic rénal impactant le pronostic vital et la stratégie thérapeutique, un dépistage des atteintes rénales au cours du syndrome de Sjögren primitif est nécessaire puisque peu de facteurs de risque ont été mis en évidence à ce jour (en dehors de la positivité des anti-SSB) [28]]. La présentation rénale la plus spécifique au cours du syndrome de Sjögren primitif est la néphropathie tubulo-interstitielle qui peut être révélée par des troubles hydroélectrolytiques isolés. Si ces troubles hydroélectrolytiques sont sévères ou associés avec un déclin (même modeste) de la fonction rénale, une biopsie rénale doit être réalisée afin de rechercher des lésions de tubulites et une néphropathie tubulo-interstitielle. Le pronostic rénal semble meilleur au cours des glomérulonéphrite membranoproliférative que des néphropathie tubulo-interstitielle sans doute parce que le diagnostic et le traitement sont réalisés à des stades plus précoces. L'association du rituximab, des stéroïdes et dans certains cas des échanges 
plasmatiques représente le traitement de choix pour les formes sévères de glomérulonéphrite membranoproliférative secondaires aux cryoglobulinémies. Pour les patients atteints de néphropathie tubulo-interstitielle, une corticothérapie (délivrant au moins $0,5 \mathrm{mg} / \mathrm{kg} / \mathrm{j}$ ) est une option efficace. La place des immunosuppresseurs ou du rituximab est débattue et des études prospectives seraient nécessaires pour juger de leur efficacité, en tant qu'épargne cortisonique ou en deuxième ligne. Le dépistage des atteintes rénales repose sur trois examens simples : créatininémie, protéinurie et ionogramme sanguin, réalisés au cours du syndrome de Sjögren primitif au moins une fois par an en cas d'atteinte systémique et deux fois par an chez les patients avec cryoglobulinémie. Des tests spécifiques sont nécessaires pour dépister les formes peu symptomatiques de néphropathie tubulointerstitielle et éviter l'insuffisance rénale chronique, puisque les traitements par stéroïdes notamment sont efficaces. La biopsie rénale doit être rapidement discutée en l'absence de cause obstructive d'insuffisance rénale (surtout en cas d'hypercalciurie) même si les patients atteints de troubles hydroélectrolytiques isolés et sans altération de la fonction rénale peuvent juste être traités de façon symptomatique. En revanche, quand une atteinte glomérulaire est suspectée, la biopsie rénale doit être rapidement pratiquée.

\section{Points essentiels}

- L'atteinte rénale est rare au cours du syndrome de Sjögren primitif et se rencontre dans moins de $10 \%$ des cas et a un pronostic le plus souvent favorable.

- La néphropathie tubulo-interstitielle est la complication rénale la plus fréquente et se caractérise par une infiltration lymphoplasmocytaire.

- Les patients souffrant d'une néphropathie tubulo-interstitielle au cours du syndrome de Sjögren primitif répondent en général bien à la corticothérapie ; la place d'autres immunosuppresseurs dont le rituximab reste à démontrer.

- Les patients avec un syndrome de Sjögren primitif et une cryoglobulinémie et une baisse du complément sont à haut risque de glomérulonéphrite membranoproliférative, et en général répondent bien aux stéroïdes, rituximab et échanges plasmatiques.

- L'atteinte rénale au cours du syndrome de Sjögren primitif est en général paucisymptomatique ; un dépistage urinaire et sanguin approprié doit donc être réalisé afin de prévenir l'insuffisance rénale chronique.

- Toute découverte d'une atteinte rénale chez un patient atteint de syndrome de Sjögren primitif doit entraîner une recherche de diagnostics différentiels comme le lupus, l'hépatite C et la maladie associée aux $\lg \mathrm{G}_{4}$. 


\section{Déclaration de liens d'intérêts}

Les auteurs déclarent ne pas avoir de liens d'intérêts en relation avec cet article.

\section{Références}

[[1] Qin B], Wang J., Yang Z., Yang M., Ma N., Huang F. Epidemiology of primary Sjögren's syndrome: a systematic review and meta-analysis. Ann Rheum Dis 2015; 74: 1983-1989.

[[2] Ramos-Casals M], Brito-Zerón P., Sisó-Almirall A., Bosch X. Primary Sjögren syndrome. Br Med J 2012; 344: e3821.

[[3] Nocturne G], Mariette X. Sjögren syndrome-associated lymphomas: an update on pathogenesis and management. Br J Haematol 2015; 168: 317-327.

[[4] Bossini N], Savoldi S., Franceschini F., Mombelloni S., Baronio M., Cavazzana I. Clinical and morphological features of kidney involvement in primary Sjögren's syndrome. Nephrol Dial Transplant 2001; 16: 2328-2336.

[[5] Thieblemont C], Berger F., Coiffier B. Mucosa-associated lymphoid tissue lymphomas. Curr Opin Oncol 1995; 7: 415-420.

[[6] Tzioufas A]G., Voulgarelis M. Update on Sjögren's syndrome autoimmune epithelitis: from classification to increased neoplasias. Best Pract Res Clin Rheumatol 2007; 21: 989-1010.

[[7] Malladi A]S., Sack K.E., Shiboski S.C., Shiboski C.H., Baer A.N., Banushree R. Primary Sjögren's syndrome as a systemic disease: a study of participants enrolled in an international Sjögren's syndrome registry. Arthritis Care Res 2012; 64: 911-918.

[[8] Baldini C], Pepe P., Quartuccio L., Priori R., Bartoloni E., Alunno A. Primary Sjögren's syndrome as a multi-organ disease: impact of the serological profile on the clinical presentation of the disease in a large cohort of Italian patients. Rheumatology 2014; 53: 839-844.

[[9] Lin D]F., Yan S.M., Zhao Y., Zhang W., Li M.T., Zeng X.F. Clinical and prognostic characteristics of 573 cases of primary Sjögren's syndrome. Chin Med J 2010; 123: 3252-3257.

[[10] Vitali C], Bombardieri S., Jonsson R., Moutsopoulos H.M., Alexander E.L., Carsons S.E. Classification criteria for Sjögren's syndrome: a revised version of the European criteria proposed by the AmericanEuropean Consensus Group. Ann Rheum Dis 2002; 61: 554-558.

[[11] Vitali C], Bombardieri S., Moutsopoulos H.M., Balestrieri G., Bencivelli W., Bernstein R.M. Preliminary criteria for the classification of Sjögren's syndrome. Results of a prospective concerted action supported by the European Community. Arthritis Rheum 1993; 36: 340-347.

[[12] Vitali C], Bombardieri S., Moutsopoulos H.M., Coll J., Gerli R., Hatron P.Y. Assessment of the European classification criteria for Sjögren's syndrome in a series of clinically defined cases: results of a prospective multicentre study. The European Study Group on Diagnostic Criteria for Sjögren's syndrome. Ann Rheum Dis 1996; 55: 116-121.

[[13] Shiboski S]C., Shiboski C.H., Criswell L.A., Baer A.N., Challacombe S., Lanfranchi H. American College of Rheumatology classification criteria for Sjögren's syndrome: a data-driven, expert consensus approach in the Sjögren's International Collaborative Clinical Alliance cohort. Arthritis Care Res 2012; 64: 475-487.

[[14] Shiboski C]H., Shiboski S.C., Seror R., Criswell L.A., Labetoulle M., Lietman T.M. 2016 American College of Rheumatology/European League Against Rheumatism classification criteria for primary 
Sjögren's syndrome A consensus and data-driven methodology involving three international patient cohorts. Ann Rheum Dis 2017; 76: 9-16.

[[15] Seror R], Ravaud P., Bowman S.J., Baron G., Tzioufas A., Theander E. EULAR Sjogren's syndrome disease activity index: development of a consensus systemic disease activity index for primary Sjogren's syndrome. Ann Rheum Dis 2010; 69: 1103-1109.

[[16] Gottenberg J]E., Seror R., Miceli-Richard C., Benessiano J., Devauchelle-Pensec V., Dieude P. Serum levels of beta2-microglobulin and free light chains of immunoglobulins are associated with systemic disease activity in primary Sjögren's syndrome. Data at enrollment in the prospective ASSESS cohort. PloS One 2013; 8: e59868.

[[17] Iwakiri D], Zhou L., Samanta M., Matsumoto M., Ebihara T., Seya T. Epstein-Barr virus (EBV)-encoded small RNA is released from EBV-infected cells and activates signaling from Toll-like receptor 3. J Exp Med 2009; 206: 2091-2099.

[[18] Nocturne G], Mariette X. Advances in understanding the pathogenesis of primary Sjögren's syndrome. Nat Rev Rheumatol 2013; 9: 544-556.

[[19] Fukatsu A], Matsuo S., Yuzawa Y., Miyai H., Futenma A., Kato K. Expression of interleukin 6 and major histocompatibility complex molecules in tubular epithelial cells of diseased human kidneys. Lab Invest 1993; 69: 58-67.

[[20] Oren R], Laufer J., Goldberg I., Kopolovic J., Waldherr R., Passwell J.H. C3, C4, factor B and HLA-DR alpha mRNA expression in renal biopsy specimens from patients with IgA nephropathy. Immunology 1995; 86: $575-583$.

[[21] Talal N], Zisman E., Schur P.H. Renal tubular acidosis, glomerulonephritis and immunologic factors in Sjögren's syndrome. Arthritis Rheum 1968; 11: 774-786.

[[22] Shearn M]A., Tu W.H. Nephrogenic diabetic insipidus and other defects of renal tubular function in Sjögren's syndrome. Am J Med 1965; 39: 312-318.

[[23] Maripuri S], Grande J.P., Osborn T.G., Fervenza F.C., Matteson E.L., Donadio J.V. Renal involvement in primary Sjögren's syndrome: a clinicopathologic study. Clin J Am Soc Nephrol 2009; 4: 1423-1431.

[[24] Goules A]V., Tatouli I.P., Moutsopoulos H.M., Tzioufas A.G. Clinically significant renal involvement in primary Sjögren's syndrome: clinical presentation and outcome. Arthritis Rheum 2013; 65: 2945-2953.

[[25] Ren H], Wang W.M., Chen X.N., Zhang W., Pan X.X., Wang X.L. Renal involvement and follow-up of 130 patients with primary Sjögren's syndrome. J Rheumatol 2008; 35: 278-284.

[[26] Cimaz R], Casadei A., Rose C., Bartunkova J., Sediva A., Falcini F. Primary Sjögren syndrome in the paediatric age: a multicentre survey. Eur J Pediatr 2003; 162: 661-665.

[[27] de Souza T]R., Silva I.H.M., Carvalho A.T., Gomes V.B., Duarte A.P., Leão J.C. Juvenile Sjögren syndrome: distinctive age, unique findings. Pediatr Dent 2012; 34: 427-430.

[[28] Jasiek M], Karras A., Le Guern V., Krastinova E., Mesbah R., Faguer S. A multicenter study of 95 biopsyproven cases of renal disease in primary Sjögren's syndrome. Rheumatology 2016;[Epub ahead of print] .

[[29] Goules A], Masouridi S., Tzioufas A.G., Ioannidis J.P., Skopouli F.N., Moutsopoulos H.M. Clinically significant and biopsy-documented renal involvement in primary Sjögren syndrome. Medicine 2000; 79: 241-249. 
[[30] Bridoux F], Kyndt X., Abou-Ayache R., Mougenot B., Baillet S., Bauwens M. Proximal tubular dysfunction in primary Sjögren's syndrome: a clinicopathological study of 2 cases. Clin Nephrol 2004; 61: 434-439.

[[31] Kim Y]K., Song H.C., Kim W.Y., Yoon H.E., Choi Y.J., Ki C.S. Acquired Gitelman syndrome in a patient with primary Sjögren syndrome. Am J Kidney Dis 2008; 52: 1163-1167.

[[32] Cohen E]P., Bastani B., Cohen M.R., Kolner S., Hemken P., Gluck S.L. Absence of H(+)-ATPase in cortical collecting tubules of a patient with Sjogren's syndrome and distal renal tubular acidosis. J Am Soc Nephrol 1992; 3: 264-271.

[[33] DeFranco P]E., Haragsim L., Schmitz P.G., Bastani B. Absence of vacuolar H(+)-ATPase pump in the collecting duct of a patient with hypokalemic distal renal tubular acidosis and Sjögren's syndrome. J Am Soc Nephrol 1995; 6: 295-301.

[[34] Inagaki Y], Jinno-Yoshida Y., Hamasaki Y., Ueki H. A novel autoantibody reactive with carbonic anhydrase in sera from patients with systemic lupus erythematosus and Sjögren's syndrome. J Dermatol Sci 1991; 2: 147-154.

[[35] Pertovaara M], Bootorabi F., Kuuslahti M., Pasternack A., Parkkila S. Novel carbonic anhydrase autoantibodies and renal manifestations in patients with primary Sjögren's syndrome. Rheumatology 2011; 50: $1453-1457$.

[[36] Takemoto F], Katori H., Sawa N., Hoshino J., Suwabe T., Sogawa Y. Induction of anti-carbonicanhydrase-II antibody causes renal tubular acidosis in a mouse model of Sjögren's syndrome. Nephron Physiol 2007; 106: 63-68.

[[37] Kim J], Tisher C.C., Linser P.J., Madsen K.M. Ultrastructural localization of carbonic anhydrase II in subpopulations of intercalated cells of the rat kidney. J Am Soc Nephrol 1990; 1: 245-256.

[[38] Pertovaara M], Korpela M., Kouri T., Pasternack A. The occurrence of renal involvement in primary Sjögren's syndrome: a study of 78 patients. Rheumatology 1999; 38: 1113-1120.

[[39] Aasarød K], Haga H.J., Berg K.J., Hammerstrøm J., Jørstad S. Renal involvement in primary Sjögren's syndrome. $Q J$ Med 2000; 93: 297-304.

[[40] Saeki T], Kawano M. IgG4-related kidney disease. Kidney Int 2014; 85: 251-257.

[[41] Mavragani C]P., Fragoulis G.E., Rontogianni D., Kanariou M., Moutsopoulos H.M. Elevated IgG Serum levels among primary Sjögren's syndrome patients: do they unmask underlying IgG4-related disease?. Arthritis Care Res 2014; 66: 773-777.

[[42] Kawano M], Suzuki Y., Yamada K., Mizushima I., Matsumura M., Nakajima K. Primary Sjögren's syndrome with chronic tubulointerstitial nephritis and lymphadenopathy mimicking $\operatorname{IgG}_{4}$-related disease. Mod Rheumatol 2015; 25: 637-641.

[[43] Nakashima Y], Nakamura H., Horai Y., Hayashi T., Takagi Y., Nakamura T. Comorbid case of IgG4related disease and primary Sjögren's syndrome. Mod Rheumatol 2015; 25: 462-467.

[[44] François H], Mariette X. Renal involvement in primary Sjögren syndrome. Nat Rev Nephrol 2016; 12: 8293.

[[45] Akiyama Y], Suzuki T., Tanaka M., Katagiri T., Ishibashi T., Imai F. A case of sarcoidosis associated with Sjögren's syndrome. Arerugī Allergy 1992; 41: 1500-1506. 
[[46] Sharma S], Gupta A., Saxena S. Comprehensive clinical approach to renal tubular acidosis. Clin Exp Nephrol 2015; 19: 556-561.

[[47] Haque S]K., Ariceta G., Batlle D. Proximal renal tubular acidosis: a not so rare disorder of multiple etiologies. Nephrol Dial Transplant 2012; 27: 4273-4287.

[[48] Fremont O]T., Chan J.C. Understanding Bartter syndrome and Gitelman syndrome. World J Pediatr 2012; 8: $25-30$.

[[49] Hinschberger O], Martzolff L., Ioannou G., Baumann D., Jaeger F., Kieffer P. Acquired Gitelman syndrome associated with Sjögren's syndrome and scleroderma. Rev Med Interne 2011; 32: e96-e98.

[[50] Casatta L], Ferraccioli G.F., Bartoli E. Hypokalaemic alkalosis, acquired Gitelman's and Bartter's syndrome in chronic sialoadenitis. Br J Rheumatol 1997; 36: 1125-1128.

[[51] Matignon M], Cacoub P., Colombat M., Saadoun D., Brocheriou I., Mougenot B. Clinical and morphologic spectrum of renal involvement in patients with mixed cryoglobulinemia without evidence of hepatitis $\mathrm{C}$ virus infection. Medicine 2009; 88: 341-348.

[[52] Terrier B], Krastinova E., Marie I., Launay D., Lacraz A., Belenotti P. Management of noninfectious mixed cryoglobulinemia vasculitis: data from 242 cases included in the CryoVas survey. Blood 2012; 119: 5996-6004.

[[53] D’Amico G], Fornasieri A. Cryoglobulinemic glomerulonephritis: a membranoproliferative glomerulonephritis induced by hepatitis C virus. Am J Kidney Dis 1995; 25: 361-369.

[[54] Cacoub P], Terrier B., Saadoun D. Hepatitis C virus-induced vasculitis: therapeutic options. Ann Rheum Dis 2014; 73: 24-30.

[[55] Mekinian A], Ravaud P., Hatron P.Y., Larroche C., Leone J., Gombert B. Efficacy of rituximab in primary Sjögren's syndrome with peripheral nervous system involvement: results from the AIR registry. Ann Rheum Dis 2012; 71: 84-87.

[[56] Terrier B], Launay D., Kaplanski G., Hot A., Larroche C., Cathébras P. Safety and efficacy of rituximab in nonviral cryoglobulinemia vasculitis: data from the French autoimmunity and rituximab registry. Arthritis Care Res 2010; 62: 1787-1795.

[[57] Gottenberg J]-E., Cinquetti G., Larroche C., Combe B., Hachulla E., Meyer O. Efficacy of rituximab in systemic manifestations of primary Sjögren's syndrome: results in 78 patients of the autoimmune and rituximab registry. Ann Rheum Dis 2013; 72: 1026-1031.

[[58] Sethi S], Fervenza F.C. Membranoproliferative glomerulonephritis--a new look at an old entity. $N$ Engl $J$ Med 2012; 366: 1119-1131.

[[59] Leadbetter E]A., Rifkin I.R., Hohlbaum A.M., Beaudette B.C., Shlomchik M.J., Marshak-Rothstein A. Chromatin-IgG complexes activate B cells by dual engagement of IgM and Toll-like receptors. Nature 2002; 416: $603-607$.

[[60] Alsuwaida A]O. Interstitial inflammation and long-term renal outcomes in lupus nephritis. Lupus 2013; 22: $1446-1454$.

[[61] Baba A], Hara S., Sato Y., Yamada K., Fujimoto S., Eto T. Three patients with nephrotic syndrome due to membranous nephropathy complicated by Sjögren's syndrome. Nihon Jinzo Gakkai Shi 2005; 47: 882-886.

[[62] Font J], Cervera R., Lopez-Soto A., Darnell A., Ingelmo M. Mixed membranous and proliferative glomerulonephritis in primary Sjögren's syndrome. Br J Rheumatol 1989; 28: 548-550. 
[[63] Siamopoulos K]C., Mavridis A.K., Elisaf M., Drosos A.A., Moutsopoulos H.M. Kidney involvement in primary Sjögren's syndrome. Scand J Rheumatol Suppl 1986; 61: 156-160.

[[64] Ronco P], Debiec H. Pathogenesis of membranous nephropathy: recent advances and future challenges. Nat Rev Nephrol 2012; 8: 203-213.

[[65] Dussol B], Tsimaratos M., Bolla G., Harlé J.R., Brunet P., Casanova P. Crescentic glomerulonephritis and primary Gougerot-Sjögren syndrome. Nephrologie 1994; 15: 295-298.

[[66] Kamachi M], Migita K., Tominaga M., Ichinose Y., Nakamura H., Origuchi T. Sjögren's syndrome complicated by MPO-ANCA positive crescentic glomerulonephritis. Nephrol Dial Transplant 1999; 14: 1033-1034.

[[67] Tatsumi H], Tateno S., Hiki Y., Kobayashi Y. Crescentic glomerulonephritis and primary Sjögren's syndrome. Nephron 2000; 86: 505-506.

[[68] Wang W]J., Wu H.S., Chu T.S. Anti-neutrophil cytoplasmic antibody-associated pauci-immune crescentic glomerulonephritis complicating Sjögren's syndrome. J Formos Med Assoc 2011; 110: 473-477.

[[69] Guellec D], Cornec-Le Gall E., Groh M., Hachulla E., Karras A., Charles P. ANCA-associated vasculitis in patients with primary Sjögren's syndrome: detailed analysis of 7 new cases and systematic literature review. Autoimmun Rev 2015; 14: 742-750.

[[70] Shen, Y., Xie J, Lin, L., Li, X., Shen, P., Pan, X., Ren, H., Chen, N. Combination Cyclophosphamide/Glucocorticoids Provide Better Tolerability and Outcomes versus Glucocorticoids Alone in Patients with Sjogren' Associated Chronic Interstitial Nephritis. Am J Nephrol 2017;46:473-480

[[71] Goules, A., Geetha, D., Arend, L. J. \& Baer, A. N. Renal involvement in primary Sjögren's syndrome: natural history and treatment outcome. Clin. Exp. Rheumatol. 37 Suppl 118, 123-132 (2019).

[[72] Kidder, D. et al. Kidney biopsy findings in primary Sjögren syndrome. Nephrol. Dial. Transplant. Off. Publ. Eur. Dial. Transpl. Assoc. - Eur. Ren. Assoc. 30, 1363-1369 (2015). 
Tableau 1A. Critères de classification diagnostiques de l'American-European Consensus Group (AECG) pour le syndrome de Sjögren [[10]].

\begin{tabular}{|l|l|}
\hline Critère & Description \\
\hline 1 & $\begin{array}{l}\text { Symptômes oculaires : réponse positive à au moins l'une des trois questions suivantes : } \\
- \text { avez-vous une sensation quotidienne d'yeux secs gênants et permanents depuis plus de } \\
\text { trois mois ? } \\
- \text { avez-vous une sensation récurrente de sable dans les yeux ? } \\
- \text { utilisez-vous des larmes artificielles plus de trois fois par jour ? }\end{array}$ \\
\hline 2 & $\begin{array}{l}\text { Symptômes buccaux : réponse positive à au moins l'une des trois questions suivantes : } \\
- \text { avez-vous une sensation quotidienne de bouche sèche depuis plus de 3 mois ? } \\
- \text { avez-vous des épisodes récidivants ou permanents de gonflement parotidien } ? \\
- \text { consommez-vous fréquemment des liquides pour avaler les aliments secs ? }\end{array}$ \\
\hline 3 & $\begin{array}{l}\text { Signes objectifs de sécheresse oculaire : au moins un des deux tests positifs : } \\
- \text { test de Schirmer } \leq 5 \mathrm{~mm} / 5 \text { min (réalisé sans anesthésie) } \\
- \text { test au rose Bengale ou autre test oculaire avec autre colorant ( } \geq 4 \text { du score de Van } \\
\text { Bijsterveld) }\end{array}$ \\
\hline 5 & $\begin{array}{l}\text { Signes objectifs de sécheresse buccale (flux salivaire } \leq 1,5 \mathrm{ml} / 15 \mathrm{~min} \text { [soit } \leq 0,1 \mathrm{ml} / \mathrm{min}]) \\
\text { et/ou sialographie parotidienne anormale, et/ou scintigraphie salivaire anormale }\end{array}$ \\
\hline 4 & $\begin{array}{l}\text { Sialadénite lymphocytaire des glandes salivaires à la biopsie des glandes salivaires } \\
\text { accessoires (focus score } \geq 1, \text { Chisholm } \geq 3 \text { ) }\end{array}$ \\
\hline 5
\end{tabular}

Le diagnostic de syndrome de Sjögren primitif repose sur : la présence d'au moins quatre de ces six critères, avec obligatoirement le critère 5 ou 6 ; la présence de trois critères parmi les quatre critères objectifs (critères 3 à 6). Le diagnostic de syndrome de Sjögren secondaire repose, chez un patient avec autre connectivite, sur: la présence des critères 1 ou 2 , en association à 2 critères (parmi critères 3 à 5). Critères d'exclusion : infection par le virus de l'immunodéficience humaine et le virus de l'hépatite $\mathrm{C}$, sarcoïdose, lymphome, radiothérapie cervicale, anticholinergiques. 
Tableau 1B. Critères de classification diagnostique de l'American-College of Rheumatology- European League Against Rheumatism (ACR-EULAR) 2016 pour le syndrome de Sjögren [[14]].

\begin{tabular}{|l|l|}
\hline Critère & Points \\
\hline $\begin{array}{l}\text { Sialadénite lymphocytaire avec focus score } \geq 1 \text { sur la biopsie de glandes } \\
\text { salivaires accessoires et focus score } \geq 1 \text { foci/4mm } \mathrm{mm}^{2}\end{array}$ & 3 \\
\hline Anticorps anti-SSA/Ro positif & 3 \\
\hline Ocular Staining Score $\geq 5$ (ou score de van Bijsterveld $\geq 4$ ) pour au moins un oeil & 1 \\
\hline Test de Schirmer $\leq 5 \mathrm{~mm} / 5 \mathrm{~min}$ pour au moins un oeil & 1 \\
\hline Flux salivaire sans stimulation $\leq 0,1 \mathrm{~mL} /$ min & 1 \\
\hline
\end{tabular}

Le diagnostic de syndrome de Sjögren primitif repose sur : la présence du critère d'inclusion (1) et de l'absence de critère d'exclusion (2) et d'un score $\geq 4$.

(1) Critère d'inclusion: applicable à tout patient ayant au moins un symptôme de sécheresse orale ou oculaire définie par une réponse positive aux question suivantes :

- Avez-vous une sensation quotidienne d'yeux secs gênants et permanents depuis au moins 3 mois?

- Avez-vous une sensation récurrente de sable dans les yeux ?

- Utilisez-vous des larmes artificielles plus de trois fois par jour?

- Avez-vous une sensation quotidienne de bouche sèche depuis au moins 3 mois?

- Consommez-vous fréquemment des liquides pour avaler les aliments secs ?

ou une suspicion de pSS selon les critères ESSDAI (1 réponse positive à au moins 1 domaine)

(2) Critères d'exclusion: antécédent d'irradiation cervicale, infection par le virus de l'immunodéficience humaine et infection active contre le virus de l'hépatite $C$, sarcoïdose, amylose, réaction du greffon contre l'hôte, maladie associée aux IgG4 
Tableau 2. Prévalence de l'atteinte rénale au cours du syndrome de Sjögren) [[7,9,15, 23,24]].

\begin{tabular}{|c|c|c|c|c|}
\hline & Pays & Critères diagnostiques & $\begin{array}{l}\text { Nombre de } \\
\text { patients }\end{array}$ & $\begin{array}{l}\text { Atteinte rénale \% } \\
\text { (n) }\end{array}$ \\
\hline Goules et al., 2000 & Grèce & European-1993 & 471 & $4,2 \%(20)$ \\
\hline Skopouli et al., 2000 & Grèce & Trois critères AECG sur six & 261 & $11 \%(30)$ \\
\hline Bossini et al., 2001 & Italie & European-1993 & 60 & $27 \%(16)$ \\
\hline $\begin{array}{l}\text { Garcia-Carrasco M et } \\
\text { al., } 2002\end{array}$ & Espagne & European-1993 & 400 & $6 \%(25)$ \\
\hline $\begin{array}{l}\text { Ramos-Casals et al., } \\
2008\end{array}$ & Espagne & AECG/European-1993 & 1010 & $5 \%(48)$ \\
\hline Maripuri et al., 2009 & États-Unis & AECG & 7276 & $0,3 \%(24)$ \\
\hline Lin et al., 2010 & Chine & AECG & 473 & $33,5 \%(192)$ \\
\hline Seror et al., 2010 & Europe & AECG & 96 & $14,58 \%(14)$ \\
\hline Malladi et al., 2012 & $\begin{array}{l}\text { Internation } \\
\text { al }\end{array}$ & AECG & 886 & $1 \%(9)$ \\
\hline Goules et al., 2013 & Grèce & AECG & 715 & $4,9 \%(35)$ \\
\hline Gottenberg et al., 2013 & France & AECG & 395 & $2,8 \%(11)$ \\
\hline $\begin{array}{l}\text { Ramos-Casals et al., } \\
2014\end{array}$ & Espagne & AECG & 921 & $4,3 \%(40)$ \\
\hline Baldini et al., 2014 & Italie & AECG/European-1993 & 1115 & $1,7 \%$ \\
\hline
\end{tabular}

AECG : American-European Consensus Criteria. 
Tableau 3. Présentation rénale spécifique au cours du syndrome de Sjögren.

Troubles hydroélectrolytiques isolés- acidose tubulaire distale avec hypercalciurie et hypocitraturie :

- diabète insipide- syndrome de Fanconi

- syndrome de Bartter

- syndrome de Gitelman

Néphrolithiase (secondaire à l'acidose tubulaire distale)

Néphrocalcinose (secondaire à l'acidose tubulaire distale)

Néphrite tubulo-interstitielle aiguë

Néphrite tubulo-interstitielle chronique

Maladie glomérulaire :

- glomérulonéphrite membranoproliférative avec ou sans cryoglobulinémie 
Tableau 4. Biopsies rénales au cours du syndrome de Sjögren (syndrome de Sjögren primitif) ${ }^{[4,23-}$ 25,28,53]].

\begin{tabular}{|c|c|c|}
\hline Biopsie rénale & $\begin{array}{l}\text { Nombre de } \\
\text { patients/nombre de } \\
\text { biopsies rénales (\%) }\end{array}$ & $\begin{array}{l}\text { Référence (classification du syndrome de } \\
\text { Sjögren primitif) }\end{array}$ \\
\hline \multirow{7}{*}{$\begin{array}{l}\text { Néphrite tubulo- } \\
\text { interstitielle }\end{array}$} & $6 / 9,66 \%$ & Bossini et al., 2001 (1993) \\
\hline & $9 / 18,50 \%$ & Goules et al., 2000 (1993) \\
\hline & $33 / 41,80,5 \%$ & Ren et al., 2008 (AECG) \\
\hline & $17 / 24,71 \%$ & Maripuri et al., 2009 (AECG) \\
\hline & $21 / 61,33 \%$ & Lin et al., 2010 (AECG) \\
\hline & $12 / 33,33 \%$ & Goules et al., 2013 (AECG) \\
\hline & $73 / 95,76,8 \%$ & Jasiek et al. (AECG et critères AECG élargis) \\
\hline \multirow{6}{*}{$\begin{array}{l}\text { Glomérulonéphrite } \\
\text { membranoproliférati } \\
\text { ve secondaire à la } \\
\text { cryoglobulinémie }\end{array}$} & $1 / 9,11 \%$ & Bossini et al., 2001 (1993)4] [29], [24], \\
\hline & $4 / 18,22 \%$ & Goules et al., 2000 (1993) \\
\hline & $2 / 41,4,9 \%$ & Ren et al., 2008 (AECG) \\
\hline & $2 / 24,8 \%$ & Maripuri et al., 2009 (AECG) \\
\hline & $10 / 33,30 \%$ & Goules et al., 2013 (AECG) \\
\hline & $8 / 95,9 \%$ & Jasiek et al. (AECG et critères AECG élargis) \\
\hline \multirow{6}{*}{$\begin{array}{l}\text { Glomérulonéphrite } \\
\text { extramembraneuse }\end{array}$} & $1 / 9,11 \%$ & Bossini et al., 2001 (1993) \\
\hline & $1 / 41,2,4 \%$ & Ren et al., 2008 (AECG) \\
\hline & $1 / 24,4 \%$ & Maripuri et al., 2009 (AECG) \\
\hline & $10 / 64,15,6 \%$ & Lin et al., 2010 (AECG) \\
\hline & $2 / 33,6 \%$ & Goules et al., 2013 (AECG) \\
\hline & $4 / 95,4 \%$ & Jasiek et al. (AECG et critères AECG élargis) \\
\hline \multirow[t]{5}{*}{ Néphropathie à IgA } & $1 / 9,11 \%$ & Bossini et al., 2001 (1993) \\
\hline & $4 / 18,22 \%$ & Goules et al., 2000 (1993) \\
\hline & $3 / 41,7,3 \%$ & Ren et al., 2008 (AECG) \\
\hline & $7 / 33,21 \%$ & Goules et al., 2013 (AECG) \\
\hline & $1 / 95,1 \%$ & Jasiek et al. (AECG et critères AECG élargis) \\
\hline \multirow{5}{*}{$\begin{array}{l}\text { Hyalinose } \\
\text { segmentaire et focale }\end{array}$} & $2 / 41,4,9 \%$ & Ren et al., 2008 (AECG) \\
\hline & $2 / 24,8 \%$ & Maripuri et al., 2009 (AECG) \\
\hline & $1 / 64,1,5 \%$ & Lin et al., 2010 (AECG) \\
\hline & $1 / 33,3 \%$ & Goules et al., 2013 (AECG) \\
\hline & $5 / 95,5 \%$ & Jasiek et al. (AECG et critères AECG élargis) \\
\hline Lésions & $1 / 24,4 \%$ & Maripuri et al., 2009 (AECG) \\
\hline
\end{tabular}




\begin{tabular}{|l|l|l|}
\hline $\begin{array}{l}\text { glomérulaires } \\
\text { minimes }\end{array}$ & $2 / 95,2 \%$ & Jasiek et al. (AECG et critères AECG élargis) \\
\hline $\begin{array}{l}\text { Glomérulonéphrite } \\
\text { proliferative non } \\
\text { spécifique }\end{array}$ & $25 / 64,39 \%$ & Lin et al., 2010 (AECG) \\
\cline { 2 - 3 } $\begin{array}{l}\text { Sclérose } \\
\text { glomérulaire }\end{array}$ & $1 / 33,3 \%$ & Goules et al., 2013 (AECG) \\
\cline { 2 - 3 } & $2 / 95,2 \%$ & Jasiek et al. (AECG et critères AECG élargis) \\
\cline { 2 - 3 } & $1 / 24,4 \%$ & Maripuri et al., 2009 (AECG) \\
\hline
\end{tabular}

AECG : American-European Consensus Criteria. 
Tableau 5. Diagnostics différentiels à évoquer en dehors du syndrome de Sjögren.

\begin{tabular}{|c|c|}
\hline Types d'atteinte rénale & Diagnostics différentiels \\
\hline $\begin{array}{l}\text { Néphropathie tubulo- } \\
\text { interstitielle }\end{array}$ & $\begin{array}{l}\text { Infection (pyélonéphrite, tuberculose, leptospirose, hantavirus) } \\
\text { Médicaments (AINS, } \beta \text {-lactamines, allopurinol) } \\
\text { Sarcoïdose } \\
\text { Maladie associée aux IgG4 } \\
\text { Syndrome TINU } \\
\text { Idiopathique } \\
\text { Hémopathie (lymphome, leucémie aiguë ou chroniques) } \\
\text { Toxique (herbes chinoises) } \\
\text { Lymphome rénal }\end{array}$ \\
\hline Acidose tubulaire distale & $\begin{array}{l}\text { Idiopathique } \\
\text { Héréditaire } \\
\text { Maladie génétique systémique (drépanocytose, Ehlers-Danlos, } \\
\text { Fabry) } \\
\text { Maladies métaboliques acquises (hyperparathyroïdie, } \\
\text { hyperthyroïdie) } \\
\text { Maladie auto-immune (lupus, cirrhose biliaire primitive, } \\
\text { hépatite auto-immune, thyroïdite) } \\
\text { Néphropathie obstructive } \\
\text { Médicaments (AINS, néphropathie aux analgésiques, lithium, } \\
\text { amphotéricine B, amiloride, ciclosporine) } \\
\text { Toxique (toluène, cyclamate) }\end{array}$ \\
\hline $\begin{array}{l}\text { Acidose tubulaire proximale } \\
\text { (syndrome de Fanconi) }\end{array}$ & $\begin{array}{l}\text { Myélome or gammapathie monoclonale } \\
\text { Médicaments (ténofovir, ifosfamide, cisplatine) } \\
\text { Toxique (plomb, cadmium) } \\
\text { Sarcoïdose } \\
\text { Maladie métabolique congénitale (maladie de Wilson, } \\
\text { cystinose, tyrosinémie, cytopathies mitochondriales) }\end{array}$ \\
\hline $\begin{array}{l}\text { Glomérulonéphrite } \\
\text { membranoproliférative } \\
\text { secondaire à la } \\
\text { cryoglobulinémie (médiée par } \\
\text { les complexes immuns) }\end{array}$ & $\begin{array}{l}\text { Lupus } \\
\text { Infection bactérienne (endocardite, infection de shunt) } \\
\text { Gammapathie monoclonale } \\
\text { Glomérulonéphrite membranoproliférative sans } \\
\text { cryoglobulinémie : } \\
\text { - infection bactérienne : endocardite, infection de shunt, } \\
\text { endocardite, mycobactéries, mycoplasme, Coxiella burnetii, }\end{array}$ \\
\hline
\end{tabular}


Brucella, nocardiose, borréliose

- infection virale : VHB, VHC, VIH

- gammapathie monoclonale

- idiopathique

AINS : anti-inflammatoires non stéroïdiens ; Ig : immunoglobulines ; TINU : tubulo-interstitial nephritis and uveitis; VHB : virus de l'hépatite B ; VHC : virus de l'hépatite C ; VIH : virus de l’immunodéficience humaine. 


\section{Légendes des figures}

Figure 1 Schéma de la physiopathologie de l'atteinte rénale au cours du syndrome de Sjögren. Auto-Ag : autoantigènes ; auto-AC : autoanticorps ; LB : lymphocytes B ; LT : lymphocytes T ; MEC : matrice extracellulaire ; IFN : interféron ; ATPase : adénosine triphosphatase ; NCCT : NaCl cotransporteur thiazide-sensible.

Figure 2 Néphrite tubulo-interstitielle chez un patient atteint de syndrome de Sjögren, trichrome de Masson.

Figure 3 Néphrite tubulo-interstitielle chez un patient atteint de syndrome de Sjögren, trichrome de Masson. Plasmocyte (flèche).

Figure 4 glomérulonéphrite membranoproliférative secondaire à la cryoglobulinémie chez un patient atteint de syndrome de Sjögren, trichrome de Masson. Cryoprécipité d'immunoglobuline (flèche).

Figure 5 glomérulonéphrite membranoproliférative secondaire à la cryoglobulinémie chez un patient atteint de syndrome de Sjögren, coloration de Marinozzi. 1. Prolifération mésangiale ; 2 doubles contours ; 3 . cryoprécipité d'immunoglobuline. 